\title{
Évaluation dosimétrique du rayonnement cosmique au dôme $C$ (Antarctique)
}

\author{
E. GAILLARD-LECANU ${ }^{1}$, F. TROMPIER ${ }^{1}$, Q. CHAU $^{1}$, \\ J.F. BOTTOLLIER-DEPOIS ${ }^{1}$, F. SPURNÝ ${ }^{2}$
}

(Manuscrit reçu le 3 décembre 2001, accepté le 9 avril 2002)

RÉSUMÉ Entre 1999 et 2001, l’Institut de protection et de sûreté nucléaire (IPSN) a réalisé des mesures de dosimétrie pour l'Institut français pour la recherche et la technologie polaire (IFRTP) afin d'évaluer la dose reçue par le personnel présent au dôme C (Antarctique), sur la future base CONCORDIA. À cet endroit, la dose est essentiellement due aux rayonnements cosmiques. Des dosimètres thermoluminescents ont été utilisés pour mesurer la contribution des rayonnements à faible transfert linéique d'énergie (TLE). La contribution à la dose des rayonnements à fort TLE a été à la fois mesurée par des détecteurs à traces et estimée par extrapolation des mesures à faible TLE, en connaissant l'altitude du dôme $\mathrm{C}$ et les proportions respectives des rayonnements à faible TLE et à fort TI.E. Les résultats globaux sont donnés en terme de dose efficace. Pour une année complète passée au dôme $C$, la dose efficace est estimée à $2,1 \mathrm{mSv}$.

ABSTRACT Dosimetry measurements of the cosmic rays carried out at the French base located at "dôme C" (Antarctic).

Between 1999 and 2001, the Institut de protection et de sûreté nucléaire (IPSN) has carried out dosimetry measurements for the Institut français pour la recherche et la technologie polaire (IFRTP), in order to estimate the dose received by the staff working at the "dôme C" (Antarctic), on the forthcoming base CONCORDIA. This dose is almost due to cosmic rays. Thermoluminescent detectors have been used to measure the part of the dose expected from particles with low energy transfer (LET). The contribution of high LET particles to the whole dose has been estimated both by direct measurements using track detectors, and from the low LET component, by extrapolation of its result. The relative contribution of low LET and high LET particles to the total dose equivalent can be deduced from the altitude of "dôme C". Results are presented in term of effective dose, which is estimated, for one year spent at "dôme C", to $2.1 \mathrm{mSv}$.

\section{Introduction}

En septembre 1999, l'Institut de protection et de sûreté nucléaire (IPSN) a été sollicité par l'Institut français pour la recherche et la technologie polaire (IFRTP)

\footnotetext{
1 Institut de radioprotection et de sûreté nucléaire, DPHD/SDOS, BP 17, 92262 Fontenay-aux-Roses Cedex, France.

${ }^{2}$ Nuclear Physics Institute of the AS CR. Na Truhlárce 39/64, 18086 Praha 8, Czech Republic.
} 


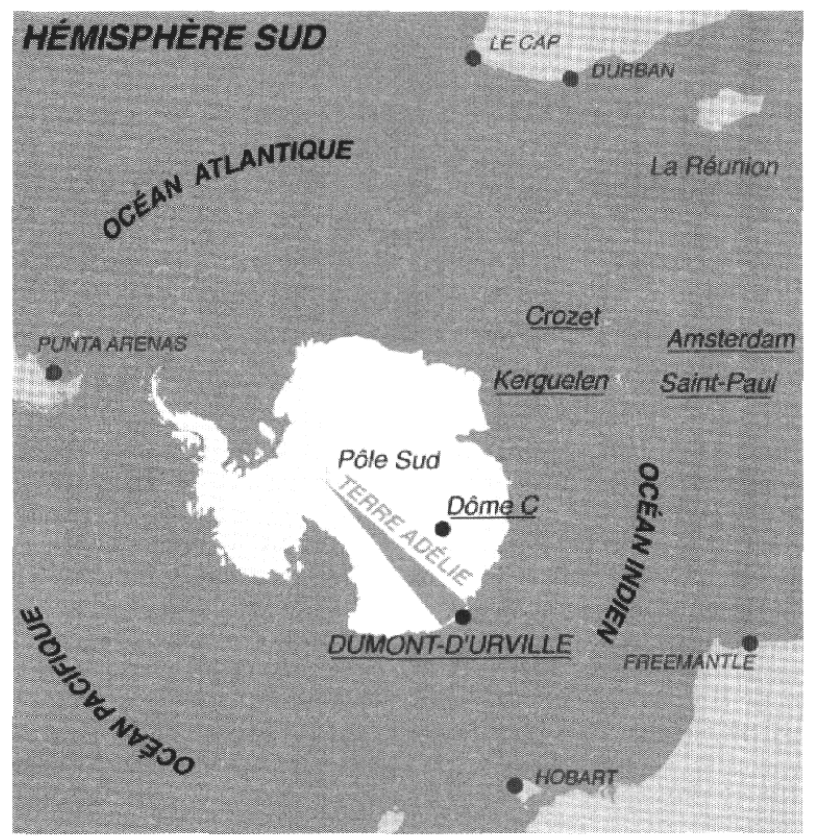

Figure 1 - Situation géographique du dôme C. Geographical situation of the "dôme C".

afin d'estimer les doses reçues par le personnel présent au dôme C (Antarctique), sur la future base CONCORDIA (Fig. 1).

Le rayonnement reçu au dôme $\mathrm{C}$ est d'origine cosmique, la composante tellurique étant considérée comme négligeable du fait de l'épaisseur importante de la calotte glacière en Antarctique. Le flux de rayonnement cosmique est plus élevé au dôme $\mathrm{C}$ que sous les latitudes tempérées. De plus, le dôme $\mathrm{C}$ se situe à l'altitude géographique de $3220 \mathrm{~m}$, ce qui correspond à une altitude équivalente de $3600 \mathrm{~m}$ en terme de pression.

Le rayonnement cosmique primaire est composé de particules chargées qui interagissent dans l'atmosphère pour créer, à l'arrivée sur Terre, un rayonnement dit secondaire qui comprend :

- des particules à faible transfert linéique d'énergie (TLE), essentiellement des photons secondaires, des électrons, des muons et les protons de plus faible énergie ;

- des particules à fort TLE dans les tissus, principalement des neutrons qui génèrent des particules secondaires par interactions. 


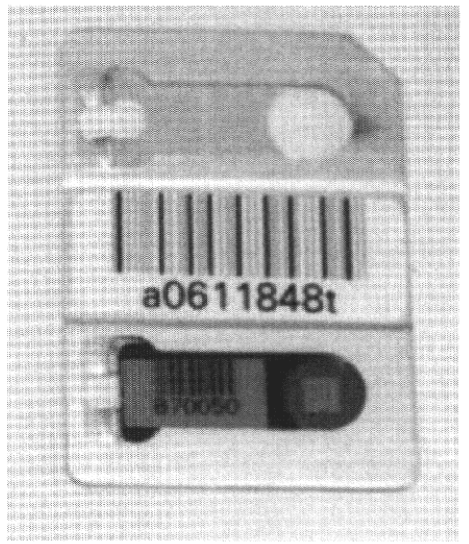

Figure 2 - Dosimètres TLD700. TLD700 dosimeters.

Pour cette étude, deux types de dosimètres ont été utilisés. La partie de l'équivalent de dose ambiant dû aux rayonnements à faible TLE est mesurée sur des dosimètres thermoluminescents (McKeever et al., 1995). L'autre partie de l'équivalent de dose ambiant est mesurée par des détecteurs à traces. Elle sera aussi estimée par calcul. Connaissant les proportions respectives des deux types de rayonnements, l'équivalent de dose ambiant dû aux rayonnements à fort TLE peut être extrapolé de la mesure issue des dosimètres thermoluminescents. À $3600 \mathrm{~m}$, la contribution à l'équivalent de dose ambiant des particules à fort TLE correspond à $30 \%$ de l'équivalent de dose ambiant total (Mc Aulay et al., 1996). Ce qui signifie que le rapport « haut TLE » sur « faible TLE » en terme d'équivalent de dose ambiant est égal à 0,43 .

Une première série de mesures a été réalisée durant les 3 mois d'été austral, de novembre 1999 à février 2000. La deuxième partie des mesures a consisté à laisser des dosimètres en place au dôme C durant un an, de novembre 1999 à février 2001.

\section{Matériel et méthodes}

Les dosimètres thermoluminescents (TLD) utilisés sont des dosimètres de type TLD700 (Fig. 2), composés de fluorure de lithium 7. Ces derniers ont fourni des résultats pour les deux campagnes de mesure. Des TLD composés d'alumine dopée au carbone ainsi que des détecteurs à traces ont complété la mesure pour la deuxième campagne.

Les TLD700 proviennent d'un lot dont la dispersion en réponse est de quelques pour-cent, et dont la stabilité temporelle a été vérifiée par des étalonnages 
successifs. Le coefficient d'étalonnage C est défini comme la dose absorbée divisée par la lecture du dosimètre en $\mathrm{nC}$, puisque le résultat de la lecture est une charge. $\mathrm{C}$ est calculé sur les étalonnages successifs réalisés sur une période de 3 ans entre 1997 et 1999 (étalonnage au ${ }^{60} \mathrm{Co}$ ).

Cinq TLD700 ont été utilisés par point de mesure. Les lots suivants ont été définis :

- un lot «témoin vol» qui a fait l'aller et le retour de Brest en Tasmanie par avion, puis a été conservé à Brest à l'IFRTP durant 6 mois jusqu'à la fin de la première série de mesures, dans des conditions de stockage satisfaisantes. Pour ce lot, il n'y a aucun séjour en Antarctique ;

- un lot « témoin IPSN » stocké à l'IPSN, au $3^{\mathrm{e}}$ étage (15 à $20 \mathrm{~m}$ de hauteur). On considère que ce lot mesure essentiellement le rayonnement cosmique ainsi que ceux issus des matériaux de construction, et non le tellurique ;

- un lot «dôme extérieur » destiné à estimer la dose reçue à l'extérieur des bâtiments ;

- un lot «dôme intérieur» destiné à estimer la dose reçue à l'intérieur des bâtiments ;

- un lot « mesure longue » resté une année entière à l'extérieur des bâtiments en Antarctique.

Le lot « témoin vol » comportait aussi plusieurs détecteurs à traces, qui ont permis de fournir une estimation de la dose « vol » pour la partie « haut TLE ».

Le lot «mesure longue » a été utilisé pour donner l'estimation de la partie « haut TLE», en employant des détecteurs à traces. De plus, une mesure complémentaire de la dose «faible TLE» a été réalisée avec des dosimètres d'alumine dopée au carbone pour ce lot.

\section{Résultats}

\subsection{Historique}

Les dosimètres ont été envoyés à Brest le 7 octobre 1999 et sont revenus à l'IPSN le 12 avril 2000, pour la première partie, et le 22 mars 2001 pour ceux qui ont séjourné plus d'un an.

D'après l'historique fourni par l'IFRTP, nous pouvons reconstituer le parcours des lots « dôme extérieur » et « dôme intérieur » entre le 7 octobre 1999 et le 12 avril 2000 :

- stockage à Brest : 66 jours,

- avion : aller et retour Paris/Tasmanie 48 heures,

- voyages hors avion : 9 jours, 
- base Dumont d'Urville (Antarctique) : 29 jours,

- dôme C : 81 jours,

soit un total de 187 jours.

Pour le lot «mesure longue », la reconstitution du parcours amène aux valeurs suivantes:

- stockage à Brest ou à l'IPSN : 59 jours,

- avion : aller et retour Paris/Tasmanie 48 heures,

- voyages hors avion : 6 jours,

- base Dumont d'Urville (Antarctique) : 27 jours,

- dôme C : 450 jours,

soit un total de 544 jours.

Pour le calcul du débit de dose au dôme $C$, la durée réelle d'exposition des dosimètres sur le site a été prise en compte. En particulier, la durée d'exposition a été corrigée des durées respectives des transports des dosimètres, que ce soit en avion ou par bateau (intitulés voyages « hors avion »). L'équivalent de dose reçu lors du voyage par avion a été évalué grâce au lot «témoin vol ». Le voyage par bateau amène une faible contribution à la dose totale mesurée, néanmoins prise en compte lors de l'évaluation de la dose attribuable à l'exposition au dôme C. En effet, seul le rayonnement cosmique au niveau de la mer doit être considéré, il n'y a pas de contribution due au rayonnement tellurique. L'estimation de l'équivalent de dose reçu pour les transferts en bateau est de 15 à $20 \mu \mathrm{Sv}$.

Dès leur retour à l'IPSN, les TLD d'alumine ainsi que les détecteurs à traces ont été renvoyés à l'Académie des sciences tchèque pour lecture.

Les TLD700 ont été lus à l'IPSN, et ont ensuite fait l'objet de plusieurs étalonnages, entre autre pour vérifier que leurs caractéristiques n'avaient pas été modifiées par les conditions de stockage, en particulier les températures extrêmes. Le tableau I montre le coefficient d'étalonnage déterminé pour la première série de dosimètres à la suite des mesures « antarctique » comparé au coefficient moyen de référence, déterminé avant ces mesures.

La sensibilité des dosimètres n'est pas affectée par les conditions particulières de température rencontrées au dôme $\mathrm{C}$.

Pour la mesure sur un an, la question du fading ${ }^{3}$ des dosimètres s'est posée. Des mesures avaient précédemment été réalisées à l'IPSN sur ces dosimètres, sur une durée de 2 mois. Aucun fading n'avait été constaté sur cette durée.

${ }^{3}$ Perte progressive de l'information initiale enregistrée au cours du temps. 


\section{TABLEAU I}

Évolution du coefficient d'étalonnage des dosimètres TLD700 avant et après les mesures Antaretique.

Variation of the calibration coefficient of the TLD700 dosimeters before and after the Antarctic measurements.

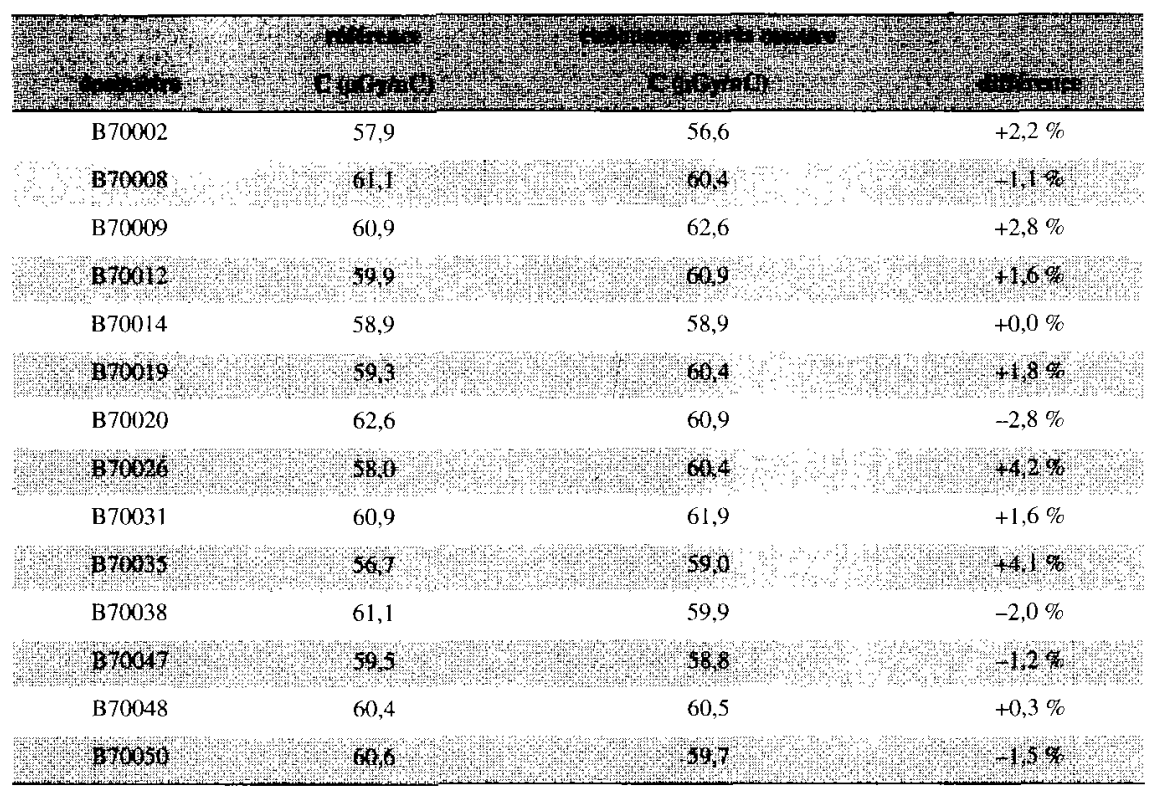

\subsection{Résultats}

\subsubsection{Partie « faible TLE» du rayonnement cosmique}

Les résultats sont donnés tout d'abord pour la partie «faible TLE» du rayonnement cosmique. Les valeurs obtenues pour le dôme $\mathrm{C}$ à l'intérieur et à l'extérieur differrent peu, la mesure à l'intérieur se situant à $7 \%$ au-dessous de la mesure à l'extérieur. Dans les résultats suivants, l'incertitude est donnée à deux écarts standards, soit un niveau de confiance de $95 \%$ :

- équivalent de dose ambiant «avion» pour l'aller et le retour France/Tasmanie : $\mathbf{8 0} \pm \mathbf{2 0} \mu \mathbf{S v}$;

- équivalent de dose ambiant «dôme $\mathrm{C} »: 270 \pm 50 \mu \mathrm{Sv}$ pour une durée de 81 jours, soit un débit d'équivalent de dose égal à $139 \pm 26 \mathrm{nSv} \mathrm{h}^{-1}$;

- équivalent de dose ambiant «bureau IPSN » : $230 \pm 35 \mu$ Sv pour une durée de 187 jours, soit un débit d'équivalent de dose égal à $52 \pm 8 \mathbf{~ n S v ~} \mathbf{~ h}^{\mathbf{- 1}}$;

- équivalent de dose ambiant « longue durée » : $1510 \pm 140 \mu \mathrm{Sv}$ pour une durée de 450 jours, soit un débit d'équivalent de dose égal à $140 \pm 13 \mathbf{n S v ~ h}^{-1}$. 
Pour la mesure « longue durée », les TLD d'alumine ont fourni un équivalent de dose ambiant pour la partie faible TLE égal à $1350 \mu \mathrm{Sv}$, tout à fait compatible avec le résultat donné par les TLD700. Les deux mesures réalisées au dôme C, sur 3 mois puis sur 1 an donnent des résultats très semblables pour les débits horaires. Ceci confirme que le fading reste négligeable pour ces dosimètres

\subsubsection{Partie " fort $T L E$ » du rayonnement cosmique}

Les mesures réalisées avec les détecteurs à traces fournissent un équivalent de dose de $1110 \pm 550 \mu \mathrm{Sv}$ pour 450 jours, ce qui donne un débit d'équivalent de dose ambiant égal à $100 \pm \mathbf{5 0} \mathbf{n S v ~ h}^{-1}$ pour la partie « haut TLE » du rayonnement cosmique. L'incertitude de $50 \%$ provient du fait que la valeur brute mesurée, comprenant le bruit de fond intrinsèque des détecteurs ainsi que la dose accumulée au dôme $\mathrm{C}$ et au cours du vol, se situe très peu au-dessus du bruit de fond intrinsèque des détecteurs (qui est de l'ordre de $1 \mathrm{mSv}$ ).

Une estimation de l'équivalent de dose ambiant «fort TLE» peut être obtenue simplement à partir de la mesure réalisée pour les particules à « faible TLE », connaissant le rapport entre les équivalents de dose ambiants à l'altitude du dôme C, pour les deux types de particules.

Au dôme C (altitude équivalente $3600 \mathrm{~m}$ ), le débit d'équivalent de dose pour la partie « haut TLE » représente $30 \%$ du débit d'équivalent de dose total. Le rapport entre la partie « haut TLE » et la partie « faible TLE » est donc 0,43 ; ce qui donne une estimation de $60 \pm 25 \mathbf{n S v} \mathbf{h}^{-1}$ pour la partie « haut TLE».

Compte tenu des différentes incertitudes, cette situation est cohérente avec les résultats de mesure.

\section{Discussion et conclusion}

Le résultat global est fourni en terme de dose efficace. Nous avons fait l'hypothèse que l'équivalent de dose ambiant est représentatif de la dose efficace. Cette approximation est valable pour la partie « faible TLE », pour laquelle l'équivalent de dose ambiant surestime légèrement la dose efficace. Pour la contribution des rayonnements cosmiques à fort TLE, qui sont, à $3600 \mathrm{~m}$ d'altitude, en partie constitués par des neutrons d'énergie élevée, cette tendance peut s'inverser et on risque alors de sous-estimer la dose efficace. Les connaissances actuelles ne permettent pas de quantifier précisément cette surestimation. Toutefois, à bord des vols commerciaux (altitude moyenne de $10 \mathrm{~km}$ ), la différence entre dose efficace et équivalent de dose ambiant est estimée à $+25 \%$ (UNSCEAR, 2000). Le dôme C se situant à une altitude plus basse, la différence entre les deux sera moins importante, et se situera de toute façon dans l'intervalle d'incertitude sur le résultat. 
Le débit de dose efficace par exposition externe due au rayonnement naturel est en moyenne de $100 \mathrm{nSv} \mathrm{h}^{-1}$ pour les zones habitées (UNSCEAR, 2000); la contribution de la composante cosmique étant de l'ordre de $45 \mathrm{nSv} \mathrm{h}^{-1}$ (Morin et al., 1999).

Le débit de dose efficace au dôme Cest estimé à $140+100=240 \mathbf{n S v ~ h}^{-1}$ pour l'ensemble des composantes du rayonnement cosmique, soit quatre à cinq fois plus que la moyenne donnée par l'Unscear pour les zones habitées.

Ceci représente une dose efficace de $0,52 \mathrm{mSv}$ pour 3 mois et de $\mathbf{2 , 1 0} \mathbf{~ m S v}$ pour l'année. L'incertitude sur cette valeur est de l'ordre de $30 \%$. À titre indicatif, l'exposition due au rayonnement naturel (cosmique + tellurique) conduit à une dose efficace annuelle de $\mathbf{0 , 8 5} \mathbf{~ m S v}$ en moyenne en Ile-de-France et $\mathbf{1 , 3 5} \mathbf{~ m S v}$ dans le Limousin.

\section{RÉFÉRENCES}

Mc Aulay R. et al. (Ed) (1996) Exposure of air crew to cosmic radiation, EURADOS report 1996-01, Bruxelles.

Mc Keever et al. (1995) Thermoluminescence dosimetry materials: properties and uses, Nuclear Technology Publishing, Ashford.

Morin A., Backe J.C., Chartier M. (1999) L'exposition externe de la population française aux rayonnements cosmiques, note technique SEGR/SAER/99-52.

UNSCEAR (2000) Sources and effects of ionizing radiation, UNSCEAR Report to the general assembly, New York. 